University of Wollongong

Research Online

Faculty of Engineering and Information

Faculty of Engineering and Information

Sciences - Papers: Part A

Sciences

$1-1-2013$

Optical vortex solitary wave in bounded nematic-liquid-crystal cell

Antonmaria A. Minzoni

Universidad Nacional Autonoma de Mexico

Luke W. Sciberras

University of Wollongong, Isciberr@uow.edu.au

Noel F. Smyth

University of Wollongong

Annette L. Worthy

University of Wollongong, annie@uow.edu.au

Follow this and additional works at: https://ro.uow.edu.au/eispapers

Part of the Engineering Commons, and the Science and Technology Studies Commons

Research Online is the open access institutional repository for the University of Wollongong. For further information contact the UOW Library: research-pubs@uow.edu.au 


\title{
Optical vortex solitary wave in bounded nematic-liquid-crystal cell
}

\begin{abstract}
Modulation theory, based on a Lagrangian formulation of the governing equations, is used to investigate the propagation of a nonlinear, nonlocal optical vortex solitary wave in a finite nematic-liquid-crystal cell. The nematic response to the vortex is calculated using the approach of themethod of images (MOI). It is demonstrated that the $\mathrm{MOI}$ is a reliable alternative to the usual Fourier series solution as it requires an order of magnitude fewer terms to obtain excellent agreement with numerical solutions. It is found that the cell walls, in addition to repelling the optical vortex solitary wave, as for an optical solitary wave, can destabilize it due to the fixed director orientation at the walls. A linearized stability analysis is used to explain and analyze this instability. In particular, the minimum distance of approach of a stable vortex to the wall is determined from the stability analysis. Good agreement is found with numerical minimum approach distances.
\end{abstract}

\section{Keywords}

nematic, bounded, cell, crystal, wave, solitary, liquid, vortex, optical

Disciplines

Engineering | Science and Technology Studies

\section{Publication Details}

Minzoni, A. A., Sciberras, L. W.., Smyth, N. F. \& Worthy, A. L. (2013). Optical vortex solitary wave in bounded nematic-liquid-crystal cell. Physical Review A: Atomic, Molecular and Optical Physics, 87 (1), 013810-1-013810-11. 


\title{
Optical vortex solitary wave in a bounded nematic-liquid-crystal cell
}

\author{
Antonmaria A. Minzoni, ${ }^{1}$ Luke W. Sciberras, ${ }^{2}$ Noel F. Smyth, ${ }^{2}$ and Annette L. Worthy ${ }^{2}$ \\ ${ }^{1}$ Fenomenos Nonlineales y Mecánica (FENOMEC), Department of Mathematics and Mechanics, Instituto de Investigación en Matemáticas \\ Aplicadas y Sistemas, Universidad Nacional Autónoma de México, 01000 México Distrito Federal, México \\ ${ }^{2}$ School of Mathematics and Applied Statistics, University of Wollongong, Northfields Avenue, Wollongong, New South Wales, Australia 2522
}

(Received 1 November 2012; published 14 January 2013)

\begin{abstract}
Modulation theory, based on a Lagrangian formulation of the governing equations, is used to investigate the propagation of a nonlinear, nonlocal optical vortex solitary wave in a finite nematic-liquid-crystal cell. The nematic response to the vortex is calculated using the approach of the method of images (MOI). It is demonstrated that the MOI is a reliable alternative to the usual Fourier series solution as it requires an order of magnitude fewer terms to obtain excellent agreement with numerical solutions. It is found that the cell walls, in addition to repelling the optical vortex solitary wave, as for an optical solitary wave, can destabilize it due to the fixed director orientation at the walls. A linearized stability analysis is used to explain and analyze this instability. In particular, the minimum distance of approach of a stable vortex to the wall is determined from the stability analysis. Good agreement is found with numerical minimum approach distances.
\end{abstract}

DOI: 10.1103/PhysRevA.87.013810

PACS number(s): 42.65.Tg, 42.70.Df, 05.45.Yv

\section{INTRODUCTION}

Optical vortices have been studied for many years, beginning with the initial theoretical work of Nye and Berry [1], who mathematically studied phase dislocations in a wave train. An optical vortex is a light beam which has an azimuthal twist, resulting in a corkscrewlike structure, such that its azimuthal phase increases by $2 n \pi$, with $n$ an integer, over one twist, with the integer $n$ being referred to as the charge of the vortex. The term vortex comes from its similarity to a vortex in fluid flow. The amplitude of the optical field is zero at the center of the vortex to adjust to the phase singularity there. It has been found that optical vortices exist in numerous media, including photorefractive lattices [2,3], rubidium vapor cells [4,5], and Bose-Einstein condensates [6]. A medium of intense interest due to its potential application in all-optical devices [7-9] is a nematic liquid crystal (NLC). The reason for this interest is that in a NLC, nonlinear effects can be observed over millimeter distances at milliwatt optical power levels due to the "huge" nonlinearity of a NLC [10]. In addition, the optical response of a NLC is termed nonlocal in that the response of the nematic extends far beyond the waist of the optical beam perturbing the nematic. This nonlocal response is vital in that it stops the usual catastrophic collapse of two-dimensional (2D) bulk solitary waves $[7,11,12]$. In addition, optical vortex solitary waves are unstable in local media, but are stable in nonlocal media, such as nematic liquid crystals, as the symmetry-breaking mode- 2 azimuthal instability is suppressed if the nonlocality is large enough $[13,14]$. In contrast to local media $[11,15]$, optical vortex solitary waves in nonlocal media have received considerably less attention, especially in a NLC [14,16-21].

In experiments, an optical vortex can be generated when a diffracting beam with a smooth wave front is input through a computer-generated holographic mask [4,22], creating a helical phase ramp whose thickness increases around the center (the singularity) of the vortex by $2 n \pi$, where $n=1,2, \ldots$. is the topological charge of the vortex [17]. A gradient of circulation is forced in the angular variable around the vortex, with the amplitude zero at the center to compensate for the phase singularity, as discussed above. Optical solitary waves, termed nematicons, and optical vortex solitary waves form in a NLC due to a balance between the diffractive spreading of an optical beam and the nonlinear self-focusing of the beam due to the nonlinear dependence of the refractive index of the nematic on the beam intensity $[7,12]$. The refractive index of the nematic changes due to the physical rotation of the threadlike nematic molecules. There is an added complication in that when the electric fields of the beam and the molecular director are orthogonal, a minimum beam power is required to rotate the molecules, known as the Freédericksz threshold $[9,10,23,24]$. To aid in overcoming this threshold so that optical beams of milliwatt powers can be used to form nematicons and optical vortex solitary waves, the nematic molecules are pretilted at an angle $\theta_{0} \sim \pi / 4$ to the optical wave front, as the Freédericksz threshold is zero when $\theta_{0}=\pi / 4$. There are two common methods to generate this pretilting, with the first being to apply an external static electric field to the liquid-crystal cell. The second method is to "rub" the cell walls, creating a static charge at the cell walls, which induces a rotation in the nematic molecules at the cell walls. This rotation then propagates into the bulk of the nematic due to intermolecular elastic forces, i.e., the nonlocality of the liquid crystal [10]. In the present paper, the second pretilt method will be used. The response of the nematic is dependent upon the chosen method of pretilt. In the case of rubbing, there is a linear (1D) or logarithmic (2D) decay of the nematic response away from the beam's center [25,26]. This implies that all of the molecules of the nematic are affected by the presence of a beam in the cell [26], so that, in particular, boundary conditions at the cell walls must be properly accounted for Refs. [25,26]. In contrast, when the first pretilt method, based on an external static electric field, is used, there is an exponential decay of the nematic response away from the beam. As a consequence, the effect of the cell walls can be neglected [27-30] for beam propagation near the center of a cell, as the cell-to-beam width ratio is around 20 to $30[31,32]$. In the nematicon case, it has been observed both experimentally [33] and theoretically in $1 \mathrm{D}[25,26]$ and 2D [34] that cell walls act repulsively towards a nematicon. 
In the present work, the behavior and propagation of an optical vortex solitary wave in a finite nematic cell is investigated. As the present work is concerned with vortex solitary waves, which are nonlinear, and not with linear optical vortices, from now on the term optical vortex will mean a vortex solitary wave. Particular attention is paid to the effect that the cell boundaries have on both the propagation and the stability of an optical vortex. To achieve this task, a blend of an exact solution found using the method of images (MOI) and a trial function, coupled with a Lagrangian formulation and modulation theory [35], is used. Modulation theory based on suitable trial functions has been found to be a useful and successful technique for giving results in excellent agreement with full numerical solutions [14,16,18,27,28,30,36] and experimental results [37,38]. In addition, it is found that the method of images possesses distinct advantages over other solution methods in that it requires an order of magnitude fewer terms to obtain accurate solutions [34]. This paper then reconfirms the usefulness of the MOI [34] in another context as a viable alternative to the standard Fourier series solution to provide evolutionary information. In addition, the MOI is used to analyze the linearized stability of an optical vortex due to its interaction with the cell walls. It is found that this interaction destabilizes the vortex if it approaches too closely to a wall. This is because the director angle is fixed at the wall due to anchoring. Previous studies have shown that an optical vortex is stable in a nematic liquid crystal due to nonlocality causing a nonzero director angle under the core of the vortex [14]. This effect cannot happen near the walls of a cell due to anchoring. A similar instability has been found when an optical vortex refracts at a nonlinear refractive index interface in a nematic liquid crystal [16], where this interface is caused by the director having two different orientations on either side of the interface [39-41]. These two instabilities are not exactly the same as the director has more freedom to rotate at the refractive index interface. The linearized stability analysis illuminates the instability mechanism and is used to determine the minimum distance of approach to a cell wall before instability occurs. These stability results are then compared with full numerical solutions of the governing equations.

\section{GOVERNING EQUATIONS}

An optical vortex is input at the NLC-air interface of a cell filled with a nematic liquid crystal. The $z$ direction is defined to be the propagation direction down the cell, with the $(x, y)$ plane orthogonal to this. The optical beam is polarized so that its electric field is in the $x$ direction. The nematic molecules have been arranged in a planar configuration in the $(x, z)$ plane. The cell walls have also undergone a pretreatment known as "rubbing," whereby a static charge is created at the cell walls, causing the molecules closest to the boundaries to rotate in the $(x, z)$ plane. Intermolecular elastic forces then propagate this director rotation into the bulk of the liquid crystal. This pretreatment results in the molecular director being prerotated in the $(x, z)$ plane at an angle $\theta_{0}$ to the $z$ direction, which helps to overcome the Freédericksz threshold [12,24], so that milliwatt power optical beams can self-focus and form nematicons or optical vortices (vortex solitary waves) with nonlinear self- focusing balancing diffractive spreading [7]. The Freédericksz threshold can be reduced to exactly zero if the pretilt angle $\theta_{0}=\pi / 4$ [42]. The optical field causes an extra rotation $\theta$ of the director, so that the total director angle is $\theta_{0}+\theta$ to the $z$ direction. For milliwatt optical beam powers, this extra rotation is small and $|\theta| \ll\left|\theta_{0}\right|$. In this small extra rotation limit, the nondimensional equations governing the propagation of the optical vortex beam in the paraxial approximation are $[25,26,33,34]$

$$
\begin{gathered}
i \frac{\partial E}{\partial z}+\frac{1}{2} \nabla^{2} E+2 \theta E=0, \\
v \nabla^{2} \theta+2|E|^{2}=0 .
\end{gathered}
$$

The Laplacian $\nabla^{2}$ is in the $(x, y)$ plane $[7,12,27] . E$ is the complex valued envelope of the electric field of the optical beam. The nonlocality parameter $v$ is related to the elastic response of the nematic and experimentally is of the order of $O(100)$ [37]. As the nematic is uniform, the walk-off angle, which is the angle between the Poynting vector and the extraordinary beam's wave vector, is also constant and so can be removed from the governing equations by a phase factor $[28,43]$, which has been done in deriving (1). The cell geometry is rectangular in the cross section, with $0 \leqslant x \leqslant L_{x}$ and $0 \leqslant y \leqslant L_{y}$. Anchoring boundary conditions give that the total director angle is fixed at the cell walls, so that $\theta=0$ at the boundaries $x=0, L_{x}$ and $y=0, L_{y}$. The nematic equations (1) and (2) are general and describe nonlinear wave propagation in many media with a diffusive response, such as photorefractive crystals [44,45] and thermal media [46-48].

The governing equations (1) and (2) have the Lagrangian

$$
L=i\left(E^{*} E_{z}-E E_{z}^{*}\right)-|\nabla E|^{2}+2 \theta|E|^{2} .
$$

The asterisk superscript denotes the complex conjugate. The linear director equation (2) can be solved exactly in terms of a Green's function $G\left(x, y, x^{\prime}, y^{\prime}\right)$, with

$$
\theta=\frac{2}{v} \int_{0}^{L_{y}} \int_{0}^{L_{x}}\left|E\left(x^{\prime}, y^{\prime}, z\right)\right|^{2} G\left(x, y, x^{\prime}, y^{\prime}\right) d x^{\prime} d y^{\prime} .
$$

\section{EVOLUTION EQUATIONS}

In general, the governing equations (1) and (2) for the evolution of a nonlinear optical beam in a nematic liquid crystal have no known exact solitary wave or vortex solutions [27]. To gain additional insights into the underlying physics and mechanics of these nonlinear beams that full numerical solutions cannot supply, averaged Lagrangian techniques [35] have proved useful, as discussed in Sec. I. The analysis presented here combines an exact solution and a trial-functionbased averaged Lagrangian method [18,26,34]. A Gaussian profile is used for the trial function of the electric field of the optical vortex and is

$$
E=\left(\operatorname{ar} e^{-r^{2} / w^{2}}+i g\right) e^{i \psi+i n \phi},
$$

where

$$
\psi=\sigma+V_{x}(x-\xi)+V_{y}(y-\eta),
$$




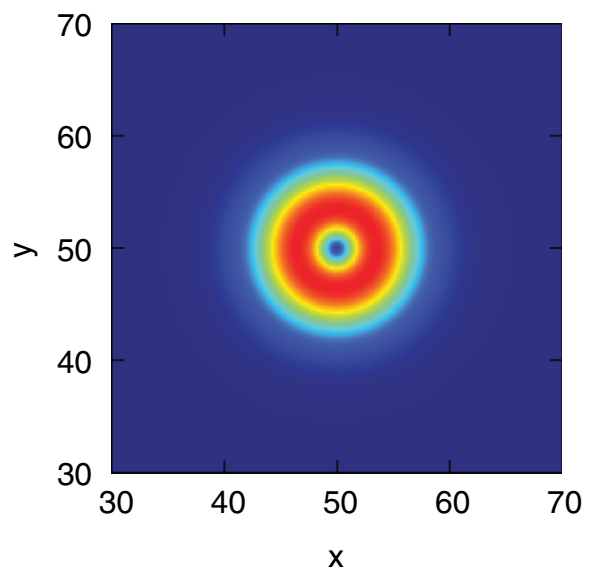

FIG. 1. (Color online) Initial profile of an optical vortex for the trial function (5) with $g=0$ and $n=1$.

and $r$ and $\phi$ are polar coordinates based on the center of the vortex,

$$
r^{2}=(x-\xi)^{2}+(y-\eta)^{2}, \quad \phi=\tan ^{-1}\left(\frac{y-\eta}{x-\xi}\right) .
$$

This trial function for the optical vortex is illustrated in Fig. 1. The first term in the trial function for the electric field is an optical vortex of amplitude $A=a w e^{-1 / 2} / \sqrt{2}$, with this peak occurring at radius $r=w / \sqrt{2}$. The vortex parameters $a$, the width $w$, the phase $\sigma$, the position $(\xi, \eta)$, the velocity $\left(V_{x}, V_{y}\right)$, and the shelf height $g$ all depend on the evolution variable $z$. The azimuthal angle is given by $\phi$ and the topological charge $n$ of the vortex gives the number of windings around the phase singularity that are undertaken by the beam. For the remainder of this paper, a charge 1 vortex is considered, so that $n=1$. The second term in the trial function is related to the shelf of low-wave-number radiation that forms under the evolving vortex [49]. This radiation can be clearly seen in Fig. 2. A perturbation analysis conducted by linearizing the electric-field equation (1) shows the existence of this shelf of radiation $[49,50]$. However, a simple physical explanation for the accumulation of the low-wave-number radiation under the vortex is that low-wave-number radiation has low group velocity, so that it cannot escape from its vicinity. Away from the vicinity of the vortex, there is linear diffractive radiation which propagates away from it. This shed radiation allows

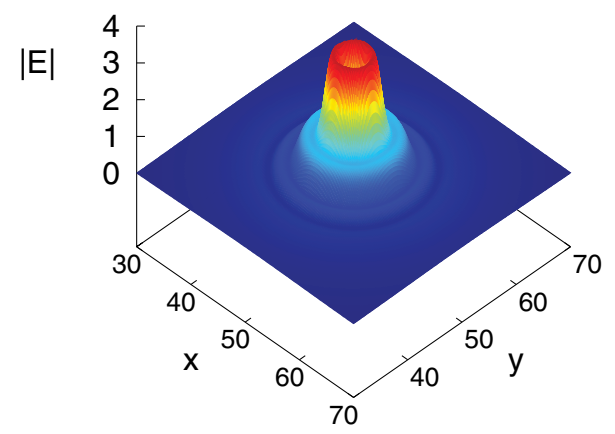

FIG. 2. (Color online) Solution for $|E|$ of the nematic equations (1) and (2) at $z=30$ for the initial condition (5) with $g=0$ showing the shelf under the vortex. the vortex to evolve to its final steady state. Finally, the shelf of low-wave-number radiation under the vortex is $\pi / 2$ out of phase with it [49]. The height of this shelf of radiation is denoted by $g$ in Eq. (5). The shelf forms under the vortex in the region $r_{\min }<r<r_{\max }$, where $r_{\max \text { min }}=w \pm R / 2$ [14].

For a nematicon, i.e., an optical solitary wave, the director perturbation can be approximated by a similar, $\operatorname{sech}^{2}$, profile as the electric field $[27,30,51]$. This is found to simulate the behavior of the liquid crystal under the influence of the beam very well. However, a similar approximation for the director does not work in the case of a vortex, as the nonlocality smoothes out its response near the central dip in the vortex so that it does not have the same profile as the electric field [14]. To overcome this difficulty, the director equation (2) is solved exactly for $\theta$ using a Green's function. The director solution $\theta$ now contains an accurate representation of the logarithmic far-field response of the nematic under the influence of the anchoring boundary conditions [25]. This use of an exact solution for the director response has been used to model nematicon propagation in both a 1D cell [26] and a 2D cell [34], with excellent comparisons with numerical solutions obtained in both cases. The MOI [52] is used here to construct a lattice of images around the point source, thus providing a solution of the director equation (2) satisfying the appropriate boundary conditions. Although a Fourier series solution of the director equation (2) can be found [34], it will not form an integral part of the analysis here, except to compare it with the solution found using the MOI and full numerical solutions. This is due to the intrinsic superiority of the MOI solution.

\section{A. Method of images}

The MOI will be employed to solve the director equation (4) [52]. A point source is then taken at $\left(x^{\prime}, y^{\prime}\right)$ within the $(x, y)$ plane of the liquid-crystal cell. An infinite series of images of this point source is then used to satisfy the boundary condition $\theta=0$ at the cell walls $x=0, L_{x}$ and $y=0, L_{y}$. This Green's function is given by Ref. [52]

$$
G=-\frac{1}{2 \pi} \operatorname{Re}\left\{\ln \left[\frac{\sigma(t-\tau, x, y) \sigma(t+\tau, x, y)}{\sigma\left(t-\tau^{*}, x, y\right) \sigma\left(t+\tau^{*}, x, y\right)}\right]\right\},
$$

where

$$
\sigma(v, x, y)=v \prod_{l \neq 0}\left[\left(1-\frac{v}{2 l}\right) e^{v /(2 l)+v^{2} /\left(8 l^{2}\right)}\right] .
$$

Here, $t$ and $\tau$ are the complex coordinates $t=x+i y$ and $\tau=x^{\prime}+i y^{\prime}$, respectively. Finally, $v$ is a complex valued and

$$
l=n L_{x}+i m L_{y}, \quad n=0, \pm 1, \ldots, \quad m=0, \pm 1, \ldots
$$

The Green's function (8) is obtained by mapping the Green's function for the half plane onto the rectangle using the elliptic function $\sigma$ [52]. The director $\theta$ is then found by substituting the Green's function (8) into Eq. (4).

The usual experimental regime for nonlinear optical beams in liquid crystals is the nonlocal one with $v$ large, i.e., $v=O(100)$ [37]. In this limit, the director distribution is slowly varying on a length scale $O(\sqrt{v})$, so that relative to the director, the vortex can be treated as a $\delta$ function. Using this approximation for the vortex, $|E|^{2}$ in the Green's function 
solution (4) gives the perturbation of the director angle as

$$
\theta=-\frac{a^{2} w^{4}}{4 v} \mathbf{R e}\left\{\ln \frac{\sigma(t-\zeta) \sigma(t+\zeta)}{\sigma\left(t-\zeta^{*}\right) \sigma\left(t+\zeta^{*}\right)}\right\},
$$

where $\zeta=\xi+i \eta$. This solution (11) for $\theta$ and the trial function (5) for the electric field $E$ are then substituted into the Lagrangian (3), which is then integrated over $(x, y)$ to obtain the averaged Lagrangian [35],

$$
\begin{aligned}
\mathcal{L}= & -\sqrt{\pi} a g^{\prime} w^{3}+\sqrt{\pi} a^{\prime} g w^{3}+3 \sqrt{\pi} a g w^{2} w^{\prime}-a^{2} w^{2} \\
& -2 g^{2} \Lambda_{2}-\frac{a^{4} w^{8}}{8 v}\left[\Delta_{1}+\Delta_{2}-\Delta_{3}-\Delta_{4}\right] \\
& -4\left(\frac{a^{2} w^{4}}{8}+g^{2} \Lambda_{1}\right)\left(\sigma^{\prime}-V_{x} \xi^{\prime}-V_{y} \eta^{\prime}+\frac{V_{x}^{2}}{2}+\frac{V_{y}^{2}}{2}\right) .
\end{aligned}
$$

Here,

$$
\begin{aligned}
\Delta_{1}= & \ln \frac{w}{\sqrt{2}}+\frac{1-\gamma}{2}-\ln 2+\ln \sqrt{\xi^{2}+\eta^{2}}-\ln (\xi \eta) \\
\Delta_{2}= & \sum_{n, m=-\infty}^{\infty}\left[\frac{1}{2} \ln \frac{\left(n L_{x}-\xi\right)^{2}+\left(m L_{y}-\eta\right)^{2}}{n^{2} L_{x}^{2}+m^{2} L_{y}^{2}}\right. \\
+ & \left.\frac{\left(\xi^{2}-\eta^{2}\right)\left(n^{2} L_{x}^{2}-m^{2} L_{y}^{2}\right)+4 n m \xi \eta L_{x} L_{y}}{2\left(n^{2} L_{x}^{2}+m^{2} L_{y}^{2}\right)^{2}}\right], \\
\Delta_{3}= & \sum_{n, m=-\infty}^{\infty}\left[\frac{1}{2} \ln \frac{n^{2} L_{x}^{2}+\left(m L_{y}-\eta\right)^{2}}{n^{2} L_{x}^{2}+m^{2} L_{y}^{2}}\right. \\
& -\frac{\left.\eta^{2}\left(n^{2} L_{x}^{2}-m^{2} L_{y}^{2}\right)\right]}{\left.2\left(n^{2} L_{x}^{2}+m^{2} L_{y}^{2}\right)^{2}\right]} \\
\Delta_{4}= & \sum_{n, m=-\infty}^{\infty}\left[\frac{1}{2} \ln \frac{\left(n L_{x}-\xi\right)^{2}+m^{2} L_{y}^{2}}{n^{2} L_{x}^{2}+m^{2} L_{y}^{2}}\right. \\
& \left.+\frac{\xi^{2}\left(n^{2} L_{x}^{2}-m^{2} L_{y}^{2}\right)}{2\left(n^{2} L_{x}^{2}+m^{2} L_{y}^{2}\right)^{2}}\right]
\end{aligned}
$$

and $\gamma$ is Euler's constant, $\gamma=0.577215665 \ldots$ Also, $\Lambda_{1}=$ $w R$ and $\Lambda_{2}=\ln \left(r_{\max } / r_{\min }\right)$. Taking variations of this averaged Lagrangian with respect to the vortex parameters gives the modulation equations describing the evolution of the vortex. The actual modulation equations are listed in Appendix A.

The final quantity to determine so that the quantities $\Lambda_{1}$ and $\Lambda_{2}$ in the averaged Lagrangian (12) are known is the radius $R$ of the shelf of low-wave-number radiation under the vortex. For a vortex in an unbounded region, it was found that $R=w[14]$. As the vortex approaches the cell walls, the shelf of radiation is affected, so that the shelf radius will not be the same as that for an unbounded region. As the actual effect of the walls on the vortex and shelf is complicated and difficult to analyze, it was found easiest to set $R=\beta w$, with $\beta$ to be determined. A comparison of solutions of the modulation equations with full numerical solutions gave that $\beta=0.2$ is robust over a wide range of input vortices. Then,

$$
\Lambda_{1}=\beta w^{2}, \quad \Lambda_{2}=\ln \left(\frac{1+\beta / 2}{1-\beta / 2}\right) .
$$

Equations governing the evolution of the vortex are found by taking variations with respect to the vortex parameters of the averaged Lagrangian (12), resulting in a system of first-order differential equations referred to as modulation equations. These are summarized in Appendix A. For comparison, Appendix B summarizes the equivalent modulation equations for when the director equation (2) is solved using a Fourier sine series. As the details of calculating these modulation equations are similar to those for the method-of-images solution, only the final modulation equations are given.

The final steady state of the vortex for a given input beam can be found from total-energy conservation for the system. This energy-conservation equation is most easily found using Nöther's theorem based on the invariance of the Lagrangian (3) with respect to shifts in $z$. Averaging this energy-conservation law by integrating in $x$ and $y$ over the cell gives the averaged energy-conservation equation,

$$
\begin{aligned}
\frac{d H}{d z} & =\frac{d}{d z} \int_{0}^{L_{y}} \int_{0}^{L_{x}}\left[|\nabla E|^{2}-2 \theta|E|^{2}\right] d x d y \\
& =\frac{d}{d z}\left\{a^{2} w^{2}+\frac{a^{4} w^{8}}{8 v}\left[\Delta_{1}+\Delta_{2}-\Delta_{3}-\Delta_{4}\right]\right\}=0 .
\end{aligned}
$$

The cell boundaries act as a repulsive force towards the vortex [14,26,34]. As a result, the vortex will traverse a spiral path inwards towards the center of the cell, where the repulsion of the boundaries is in balance. Hence, at the steady state, the vortex will be located at the center of the cell. Let us denote steady-state values of quantities by a carat superscript. We then have $\hat{\xi}=L_{x} / 2$ and $\hat{\eta}=L_{y} / 2$ with $\hat{V}_{x}=0$ and $\hat{V}_{y}=0$. Furthermore, at the steady state, the vortex will no longer shed radiation and hence the shelf height $\hat{g}$ will be zero. Thus, the modulation equation (A6) can be used to find the steady-state relationship between $\hat{a}$ and $\hat{w}$, which is

$$
\hat{a}^{2}=\frac{16 v}{\hat{w}^{6}} \text {. }
$$

The combination of Eqs. (18) and (19) is used to determine the final steady-state values of the amplitude $\hat{a}$ and width $\hat{w}$ for a given initial condition.

\section{B. Stability analysis}

Vortices are unstable in local media [2,3,11,53]. However, vortices in nonlocal media are stable for high enough nonlocality $[13,14]$. These results are for a vortex propagating in an infinite region. We shall now study how the interaction of a vortex with a boundary can change its stability, even in a nonlocal medium for which the nonlocality is high enough to guarantee stability away from the boundary. This linearized stability analysis will be based on the method of images.

To simplify the stability analysis, let us assume that the vortex is close enough to one wall so that the effect of the other three walls can be neglected. Let us take this wall to be the plane $x=0$. To calculate the corresponding Green's function for this simplified geometry, a point source $\left(x^{\prime}, y^{\prime}\right)$ is taken within the half plane, $x>0$. The boundary condition on the wall is satisfied by taking a point sink at the image point of the source $\left(-x^{\prime}, y^{\prime}\right)$. Using this new Green's function, an appropriate 
averaged Lagrangian can be calculated from Eqs. (3) and (4). However, the calculation of this averaged Lagrangian differs in one major aspect from that of the previous section. The stability of the vortex is dependent on the azimuthal angle $\phi$ around the vortex as a vortex becomes unstable via a symmetry-breaking azimuthal instability which splits the vortex in two $[13,14]$. To account for this, the vortex parameters need to be functions of both $z$ and $\phi$ in the stability analysis.

Therefore, the Green's function for the half plane with zero boundary condition on the plane is

$$
\begin{aligned}
G= & -\frac{1}{2 \pi}\left[\ln \sqrt{\left(x-x^{\prime}\right)^{2}+\left(y-y^{\prime}\right)^{2}}\right. \\
& \left.-\ln \sqrt{\left(x+x^{\prime}\right)^{2}+\left(y-y^{\prime}\right)^{2}}\right] .
\end{aligned}
$$

The trial function for the electric field $E$ remains (5), but now with all parameters depending on $z$ and $\phi$. By substituting (20) and the trial function (5) for the electric field $E$ into the Green's function solution (4) for $\theta$, and using the previously discussed asymptotic $\delta$ function approximation valid in the nonlocal limit to evaluate the resulting integral, the director angle for the half plane is

$$
\theta=-\frac{a^{2} w^{4}}{4 v}\left[\ln \frac{w}{\sqrt{2}}+\frac{1-\gamma}{2}-\ln \sqrt{(x+\xi)^{2}+(y-\eta)^{2}}\right] .
$$

Due to the new dependence of the parameters on the polar angle $\phi$, the Lagrangian for the nematic equations (1) and (2) is

$$
L=i\left(E^{*} E_{z}-E E_{z}^{*}\right)-\left|E_{r}\right|^{2}-\frac{\left|E_{\phi}\right|^{2}}{r^{2}}+2 \theta|E|^{2} .
$$

The averaged Lagrangian is then found by substituting the trial function $E$ (5) and the exact solution for $\theta(21)$ in the half plane into the Lagrangian (22) and averaging in $x$ and $y$ by integrating over the half plane $x>0$, resulting in

$$
\begin{aligned}
\mathcal{L}= & -\sqrt{\pi} a g_{z} w^{3}+\sqrt{\pi} a_{z} g w^{3}+3 \sqrt{\pi} a g w^{2} w_{z} \\
& +2 \sqrt{\pi} a_{\phi} g w+2 \sqrt{\pi} a g w_{\phi}-2 \sqrt{\pi} a g_{\phi} w \\
& -4\left(\frac{a^{2} w^{4}}{8}+g^{2} \Lambda_{1}\right)\left(\sigma_{z}-V_{x} \xi_{z}-V_{y} \eta_{z}+\frac{V_{x}}{2}+\frac{V_{y}}{2}\right) \\
& -a^{2} w^{2}-\frac{a_{\phi}^{2} w^{2}}{2}-a^{2} w_{\phi}^{2}-2 g^{2} \Lambda_{2}-a w a_{\phi} w_{\phi}-2 g_{\phi}^{2} \Lambda_{2} \\
& -\frac{a^{4} w^{8}}{8 v}\left(\ln \frac{w}{\sqrt{2}}+\frac{1-\gamma}{2}-\ln 2 \xi\right) .
\end{aligned}
$$

It is noted that the phase $\sigma$ is taken to be a function of $z$ alone. This is because $w$ and $g$ are the conjugate variables governing the stability of the vortex, so that taking $\sigma$ not to be a function of $\phi$ does not affect the linearized stability analysis [14]. The equations for the shelf of radiation given by Eq. (17) remain valid. The resulting modulation equations are given in Appendix C.

A linearized stability analysis about the steady state is now conducted to find the minimum distance of approach of the vortex to the boundary before it becomes unstable. We perturb about the steady state with $a=\hat{a}+a_{1}, w=\hat{w}+w_{1}$, and $g=$ $\hat{g}+g_{1}=g_{1}$, as $\hat{g}=0$. The modulation equations (C1), (C2), and (C6) are linearized using the above perturbations, with modal solutions of the form

$$
w_{1}=W e^{i(\lambda z+\ell \phi)}, \quad g_{1}=G e^{i(\lambda z+\ell \phi)}
$$

sought. The linearized mass equation $(\mathrm{C} 1)$ gives

$$
a_{1}=-\frac{2 \hat{a}}{\hat{w}} w_{1} \text {. }
$$

After some algebra, the eigenvalues $\lambda$ are found to be

$$
\lambda=\frac{4 \pi \ell \pm \sqrt{-8 \pi \hat{w}^{2} M_{1} M_{2}}}{2 \pi \hat{w}^{2}},
$$

where

$$
\begin{gathered}
M_{1}=\frac{3}{\hat{w}^{2}}+\frac{\ell^{2}}{\hat{w}^{2}}-\frac{\hat{a}^{2} \hat{w}^{4}}{16 v}, \\
M_{2}=\frac{4 \Lambda_{1}}{\hat{w}^{2}}+\frac{\hat{a}^{2} \hat{w}^{4} \Lambda_{1} K_{1}}{v}-\left(1+\ell^{2}\right) \Lambda_{2}, \\
K_{1}=\ln \frac{\hat{w}}{\sqrt{2}}+\frac{1-\gamma}{2}-\ln (2 \xi) .
\end{gathered}
$$

The vortex is then unstable when $M_{1} M_{2}>0$, with the borderline case being when $M_{2}=0$. We thus determine the minimum distance of approach of the vortex to the wall at which instability sets in as

$$
\xi_{\min }=\frac{1}{2} e^{-K},
$$

where

$$
K=\frac{v}{\hat{a}^{2} \hat{w}^{4} \Lambda_{1}}\left[-\frac{4 \Lambda_{1}}{\hat{w}^{2}}+\left(\ell^{2}+1\right) \Lambda_{2}\right]-\ln \frac{\hat{w}}{\sqrt{2}}-\frac{1-\gamma}{2} .
$$

The vortex is stable for distances $\xi$ for which $M_{1} M_{2}<0$. Here, $\ell$ is the mode number of the eigenmode. It has been previously found that the most unstable mode in an infinite region occurs when $\ell=2[13,14]$. The expression (26) shows that this is also true for the present finite nematic cell in that $\operatorname{Im}(\lambda)$ is maximum when $\ell=2$ for $M_{1} M_{2}>0$, in agreement with numerical solutions which show that the vortex splits into two beams when it becomes unstable on approach to a wall, as will be discussed in the next section. In addition, this result that $\ell=2$ is the most unstable mode is insensitive to the value of $\beta$. Hence, $\ell=2$ will be used in Eq. (30) for comparison with numerical solutions in the next section. It should be noted that the above stability analysis has assumed that the distance $\xi$ is fixed in the trial function (5). If the distance is also allowed to vary, then the stability analysis becomes much more involved and no simple formula equivalent to Eq. (30) can be found. Numerical solutions show that the vortex becomes highly distorted as it approaches the wall, as would be expected, so that fixed trial functions for its profile cease to be valid. So even including the distance perturbation will not capture the full dynamical process. The expression (30) will be found to be in reasonable accord with numerical solutions, even given the approximations involved, and so represents a balance between simplicity and capturing the full details.

\section{RESULTS AND DISCUSSION}

In this section, we will compare the results found from full numerical solutions of the governing equations (1) and (2) with those of the modulation equations found by using both the method-of-images and the Fourier series solution of 
the director equation (2). The modulation equations, given in Appendices A and B, were solved using the standard fourth-order Runge-Kutta method. The full numerical solution of the electric-field equation (1) was found using second-order centered differences for the Laplacian $\nabla^{2} E$ and a secondorder predictor-corrector method, based on the second-order Runge-Kutta method, to advance forward in $z$, which is the propagation direction. The director equation (2) was solved using centered second-order differences for the Laplacian $\nabla^{2} \theta$ and Jacobi iteration to solve the resulting linear system. The initial condition for the optical beam envelope for the numerical solutions is the trial function (5) with the shelf height $g$ equal to zero, as the vortex has not started to shed radiation. To satisfy the stability criteria and maintain accuracy, the step sizes used were $\Delta x=\Delta y=0.2$ and $\Delta z=0.001$. The propagation length used was $z=500$, which is a typical nondimensional cell length [37]. The numerical investigation for the propagation of an optical vortex was conducted within a square NLC cell with nondimensional width and breadth $\left(L_{x}, L_{y}\right)=(100,100)$.

Let us first consider the vortex propagating sufficiently far from the cell walls so that it does not become unstable. We shall also compare and contrast the behavior of an optical vortex with a nematicon in the same cell [34]. An optical vortex shows the same "bouncing" as a nematicon, for the same reasons as for a nematicon [33,34]. The vortex is also stable if it is sufficiently far from a wall, as found for a vortex in a circular cell $[17,18]$. The parameter values were chosen so that the vortex solitary wave is above the minimum power threshold for its existence and below the threshold at which it will split into two. Figure 3 shows a typical comparison between the full numerical solution and solutions of both sets of modulation equations. Previous work has compared experimental data, full numerical solutions, and the first harmonic mode of a Fourier series solution for the trajectory of a nematicon, with the first harmonic giving an adequate approximation [54]. This idea was taken further with the method-of-images solution with only the fundamental images neighboring the physical cell used to calculate the trajectory, with very good results obtained [34]. The same idea will be used here for the vortex, with the first eight images used to approximate its evolution, with an obvious advantage in the amount of calculation required. Figure 3(a) shows the comparison for the amplitude $A$ of the vortex. It can be seen that the first eight images are all that is needed to obtain good agreement with the numerical solution, with no discernible difference from taking 10200 images. The Fourier series modulation solution shows a period difference with both the full numerical and method-of-images solution, with the periods of the latter two in agreement. The method-of-images and Fourier modulation solutions have different periods as different approximations have been used to calculate the corresponding modulation equations. The numerical amplitude shows more complicated behavior than the modulation solutions. This is due to the vortex becoming distorted as it approaches the cell walls, as can be seen when comparing the numerical amplitude shown in Fig. 3(a) with the position shown in Fig. 3(d). These distortions cannot be captured by the fixed trial function (5).

Figures 3(b) and 3(c) show comparisons for the trajectory components $\xi$ and $\eta$ as functions of $z$, respectively. It can be
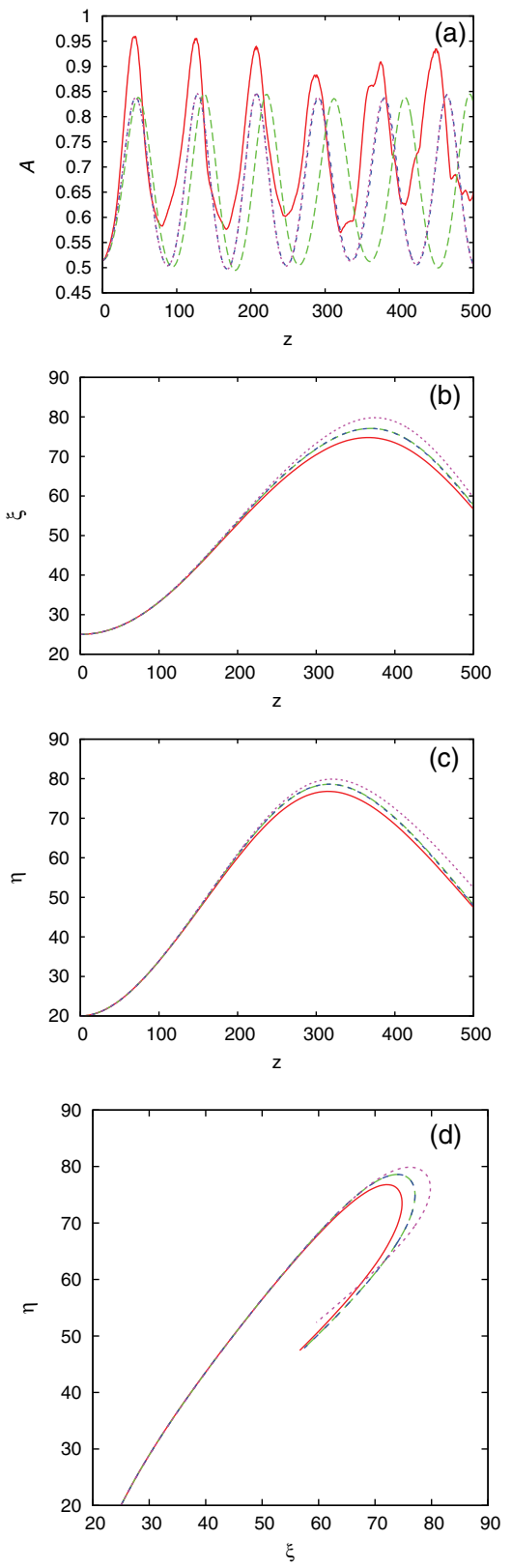

FIG. 3. (Color online) Comparison between the full numerical solution (red solid line), Fourier series solution (green dashed line), MOI solution (blue dot-dashed line), and MOI solution including only the first eight images (magenta dotted line) for (a) amplitude $A$, (b) $x$ position, (c) $y$ position, and (d) $(x, y)$ position for a square cell. The initial values are $a=0.15, A=0.52 \ldots, w=8,(\xi, \eta)=(25,20)$, and $\left(V_{x}, V_{y}\right)=(0,0)$, with $v=200$ and $\left(L_{x}, L_{y}\right)=(100,100)$.

seen that both modulation solutions give identical results that are in excellent agreement with the full numerical trajectory. This is because the amplitude-width and position-velocity oscillations of nonlinear beams in liquid crystals tend to decouple $[28,36,55,56]$, so even though the method-of-images and Fourier series solutions give different amplitudes, they can agree in the position. Again, the first eight nearest-neighbor images give a very good comparison with the numerical position. This further confirms the superiority of the MOI over traditional Fourier series solutions, as first suggested in 


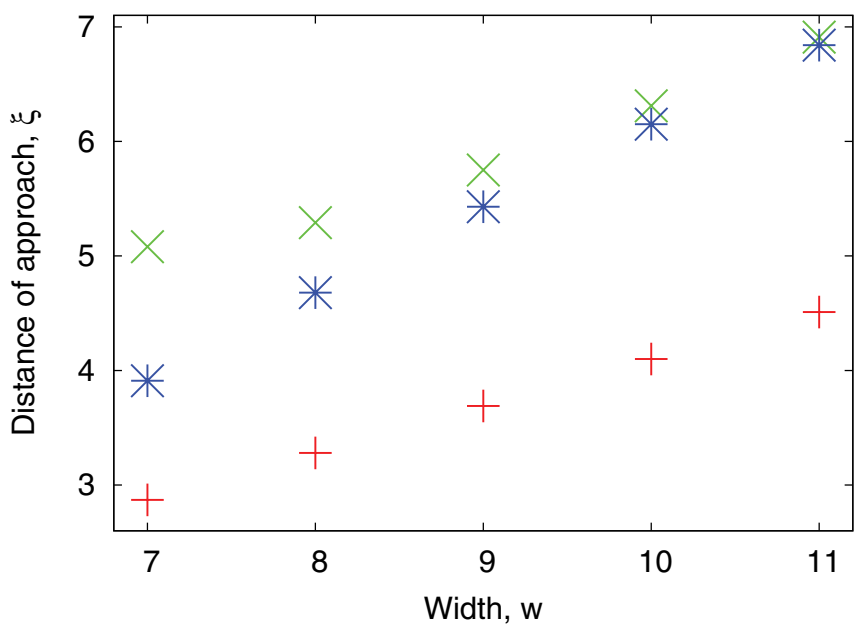

FIG. 4. (Color online) A comparison between the analytical stability boundary (30) (red plus sign, + ) with the full numerical solution (green crosses, $\times)$ for $\left(V_{x}, V_{y}\right)=(0.8,0)$ and the full numerical solution (blue stars, *) for $\left(V_{x}, V_{y}\right)=(1.5,0)$. The numerical stability boundary is the distance from the boundary at which instability first occurs. The initial conditions used were $a$ given by Eq. (32), $(\xi, \eta)=(50,100)$ for $\left(L_{x}, L_{y}\right)=(100,200)$.

Ref. [34]. Taking the $z$ direction to be into the page, the final plot [Fig. 3(d)] shows the helical trajectory of the vortex as it propagates down the liquid-crystal cell. The repulsive nature of the cell walls can be clearly seen. As the vortex was not given an initial velocity, the vortex's initial motion arises from this repulsive force. Thus, if the interaction with the boundary does not disturb the phase singularity at the center of the vortex, the cell walls will completely repel the vortex and stability will be maintained, as in a circular cell [17,18]. Figure 3(d) confirms the conclusions drawn from Figs. 3(b) and 3(c), i.e., both modulation solutions are in agreement and are in excellent agreement with the numerical solution, and the first eight nearest-neighbor images give an excellent approximation to the vortex trajectory.

A comparison between the analytical minimum distance of approach for stability (30) and this minimum distance as given by numerical solutions is shown in Fig. 4. This stability boundary is shown as a function of the initial width $w$ of the vortex for two different initial velocities, $\left(V_{x}, V_{y}\right)=$ $(0.8,0)$ and $\left(V_{x}, V_{y}\right)=(1.5,0)$. The stability boundary (30) was obtained using a small perturbation from the steady vortex. Hence, to obtain a comparison with numerical solutions, the numerical initial condition must also be near a steady vortex. Equation (C6) of Appendix $\mathrm{C}$ gives the amplitude-width relation for the steady vortex as

$$
a^{2} w^{6}=16 v .
$$

This equation was used to determine the initial amplitude for a given initial vortex width for the comparisons of Fig. 4. The cell dimensions were chosen to be large enough so that the vortex was concentrated in the vicinity of one wall only. The analytical and numerical minimum distances of approach are in reasonable agreement for all widths, but for a higher initial velocity, the smaller vortex widths are in better agreement. However, Fig. 4 shows that the assumptions used in Sec. III B
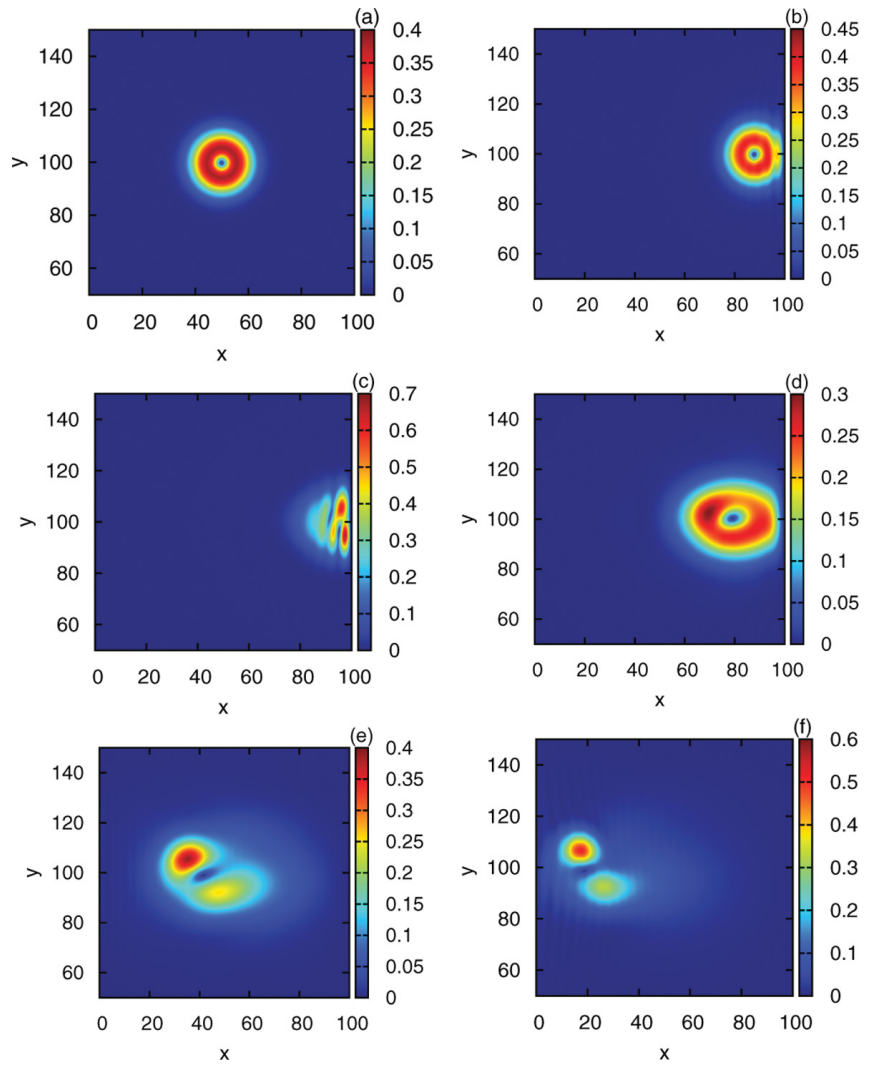

FIG. 5. (Color online) A sequence showing a vortex colliding with a cell wall, showing the instability of the vortex. (a) $z=0$, (b) $z=50$, (c) $z=75$, (d) $z=100$, (e) $z=150$, (f) $z=180$. The initial vortex parameters are $w=8$, with $a$ given by Eq. (32), $\xi=$ 50, $\eta=100, V_{x}=0.8$, and $V_{y}=0$, with $v=200, L_{x}=100$, and $L_{y}=200$.

for the linearized stability analysis are good approximations. The numerical solutions show that the minimum distance of approach for stability is only weakly dependent on the initial velocity, particularly at larger initial widths. The differences between the minimum distances of approach as given by the linearized stability analysis and the numerical solutions are mainly due to the distortion of the vortex as it approaches the cell wall, with the leading edge of the vortex being squashed against the wall. The trial function $E$, given by Eq. (5), does not take into account any deformations of the vortex, other than changes in its amplitude and width for a fixed functional form, during interaction with the boundary.

Figure 5 shows the numerical evolution of the vortex for a typical case for which the vortex approaches closer than the minimum distance and is reflected, breaking up into two nematicons. The large deformation of the vortex can be clearly seen, particularly at its leading edge in Fig. 5(c). The vortex becomes unstable due to the anchoring boundary condition fixing $\theta=0$ at the wall. Nonlocality stabilizes an optical vortex in a liquid crystal due to the nonlocality lifting the director angle $\theta$ sufficiently above zero under the core of the vortex [14]. This cannot occur near the boundary, so that the vortex is behaving like one in a local medium, for which vortices are unstable to a mode- 2 symmetry-breaking azimuthal instability [13], noting that in a local medium the director perturbation 
is also zero at the vortex center. A further complication in comparing the analytical formula and numerical solutions is that the numerical vortex position is calculated as the vortex's center of mass, that is,

$$
\xi=\frac{\int_{0}^{L_{y}} \int_{0}^{L_{x}} x|E|^{2} d x d y}{\int_{0}^{L_{y}} \int_{0}^{L_{x}}|E|^{2} d x d y} .
$$

This is clearly the center (phase singularity) of the vortex when it is symmetric. Figure 5 shows that interaction with the boundary distorts the vortex, so that its center of mass will not coincide with its phase singularity. This accounts for some of the differences between the analytical formula (30) and numerical solutions seen in Fig. 4. Figure 5(f) shows that when the vortex splits into nematicons, the nematicons do not separate, but are bound together, unlike in local media $[4,11]$. This is due to nonlocality as the nematic response extends far beyond the optical beams and acts as an attractive potential. This attraction of nonlinear beams in (nonlocal) liquid crystals is in accord with experimental observations $[19,20]$ and theoretical results [29,57].

\section{CONCLUSIONS}

The propagation of an optical vortex in a finite liquidcrystal cell has been studied. A Lagrangian formulation of the governing equations was used in conjunction with a hybrid of an exact solution and a trial function to obtain modulation equations for the vortex evolution. The exact solution of the director equation was found using both the method-of-images and a standard Fourier series solution. Both methods produced modulation equations whose solutions were in good agreement with numerical solutions, but the method-of-images solution was found to be much more efficient as only the eight nearestneighbor images are required to give this good agreement. This confirms the prediction given in Ref. [34] that the MOI is a powerful tool and an excellent alternative to Fourier series solutions for the director distribution. There is scope for its application to other nonlinear beam evolution scenarios in bulk media. Furthermore, the MOI can be applied to any number of cell geometries for which the Green's function for the director equation can be found in closed form using conformal mapping, as for the present case in which the Green's function was found by a mapping of the half plane onto a rectangle. The MOI solutions were compared with solutions obtained using a Fourier series solution of the director equation to emphasize the efficiency of the MOI in solving the director equation.

The method of images was also used to analyze the stability of the vortex in a cell, in particular the effect of the anchoring boundary condition on the vortex stability. To simplify this analysis, the vortex was assumed to be close enough to one boundary so that the effect of the other boundaries could be ignored. This assumption enabled an analytical expression for the closest distance of approach of the vortex to a boundary before instability set in to be obtained. This closest-approach formula was found to be in reasonable agreement with numerical results, especially as numerical solutions showed that the vortex becomes highly distorted as it approaches a boundary. This simple use of the method of images reinforces its utility in nonlinear beam propagation problems. Future work will consider other cell geometries.

\section{ACKNOWLEDGMENT}

The authors wish to acknowledge the detailed and perceptive comments and suggestions of the anonymous referee, which helped to greatly improve the presentation of this work.

\section{APPENDIX A: MODULATION EQUATIONS: METHOD OF IMAGES}

Taking variations of the averaged Lagrangian (12) with respect to the vortex parameters gives the modulation equations for the evolution of the vortex as

$$
\begin{aligned}
& \frac{d}{d z}\left[\frac{a^{2} w^{4}}{8}+g^{2} \Lambda_{1}\right]=0 \\
& \sqrt{\pi} \frac{d}{d z}\left(a w^{3}\right)-4 g \Lambda_{1} \frac{d \sigma}{d z}=-2 g \Lambda_{1}\left(V_{x}^{2}+V_{y}^{2}\right)+2 g \Lambda_{2}, \\
& \xi^{\prime}=V_{x}, \quad \eta^{\prime}=V_{y}, \\
& \frac{d}{d z}\left[\left(\frac{a^{2} w^{4}}{8}+g^{2} \Lambda_{1}\right) V_{x}\right] \\
& =\frac{a^{4} w^{8} \eta^{2}}{32 \nu \xi\left(\xi^{2}+\eta^{2}\right)}+\frac{a^{4} w^{8}}{32 \nu} \sum_{n, m=-\infty}^{\infty}\left[\frac{\left(n L_{x}-\xi\right)}{\left(n L_{x}-\xi\right)^{2}+\left(m L_{y}-\eta\right)^{2}}-\frac{2 n m \eta L_{x} L_{y}}{\left(n^{2} L_{x}^{2}+m^{2} L_{y}^{2}\right)^{2}}-\frac{\left(n L_{x}-\xi\right)}{\left(n L_{x}-\xi\right)^{2}+m^{2} L_{y}^{2}}\right], \\
& \frac{d}{d z}\left[\left(\frac{a^{2} w^{4}}{8}+g^{2} \Lambda_{1}\right) V_{y}\right] \\
& =\frac{a^{4} w^{8} \xi^{2}}{32 v \eta\left(\xi^{2}+\eta^{2}\right)}+\frac{a^{4} w^{8}}{32 v} \sum_{n, m=-\infty}^{\infty}\left[\frac{\left(m L_{y}-\eta\right)}{\left(n L_{x}-\xi\right)^{2}+\left(m L_{y}-\eta\right)^{2}}-\frac{2 n m \xi L_{x} L_{y}}{\left(n^{2} L_{x}^{2}+m^{2} L_{y}^{2}\right)^{2}}-\frac{\left(m L_{y}-\eta\right)}{n^{2} L_{x}^{2}+\left(m L_{y}-\eta\right)^{2}}\right],
\end{aligned}
$$




$$
\begin{gathered}
\frac{d g}{d z}=\frac{a}{\sqrt{\pi} w}-\frac{a^{3} w^{5}}{16 \sqrt{\pi} v}, \\
\frac{d \sigma}{d z}=\frac{1}{2}\left(V_{x}^{2}+V_{y}^{2}\right)-\frac{4}{w^{2}}-\frac{a^{2} w^{4}}{2 v}\left[\Delta_{1}+\Delta_{2}-\Delta_{3}-\Delta_{4}-\frac{1}{4}\right] .
\end{gathered}
$$

The modulation equation (A1) is the equation for conservation of mass (optical power), and Eqs. (A4) and (A5) are those for conservation of $x$ and $y$ momentum, respectively. The primary concern of the present work is the trajectory of the vortex, which is given by the modulation equations (A3), (A4), and (A5).

As the vortex evolves, it sheds diffractive radiation in order to settle to a steady state [27,49,51]. The flux of diffractive radiation from a nematicon has been calculated previously [27,49,51], but since the linearized equations governing this radiation are the same for a nematicon and a vortex, the same radiative loss results hold. This previous radiation analysis shows that when radiative loss is included in the modulation equations, the mass equation (A1) and the modulation equation for $g$ (A6) become

$$
\frac{d}{d z}\left[\frac{a^{2} w^{4}}{8}+g^{2} \Lambda_{1}\right]=-2 \delta \tilde{\Lambda} \kappa^{2}
$$

and

$$
\frac{d g}{d z}=\frac{a}{\sqrt{\pi} w}-\frac{a^{3} w^{5}}{16 \sqrt{\pi} v}-2 \delta g
$$

respectively. The loss coefficient $\delta$ is given by

$$
\delta=-\frac{\sqrt{2 \pi}}{32 e \kappa \tilde{\Lambda}} \int_{0}^{z} \pi \kappa\left(z^{\prime}\right) \ln \left[\left(z-z^{\prime}\right) / \tilde{\Lambda}\right]\left[\left(\left\{\frac{1}{2} \ln \left[\left(z-z^{\prime}\right) / \tilde{\Lambda}\right]\right\}^{2}+\frac{3 \pi^{2}}{16}\right)^{2}+\frac{\pi^{2}}{16}\left\{\ln \left[\left(z-z^{\prime}\right) / \tilde{\Lambda}\right]\right\}^{2}\right]^{-1} \frac{d z^{\prime}}{\left(z-z^{\prime}\right)},
$$

where

$$
\kappa^{2}=\frac{1}{\tilde{\Lambda}}\left[\frac{1}{8} a^{2} w^{4}-\frac{1}{8} \hat{a}^{2} \hat{w}^{4}+\tilde{\Lambda} g^{2}\right] .
$$

It should be noted that there was a misplaced bracket in the term $\left\{\frac{1}{2} \ln \left[\left(z-z^{\prime}\right) / \tilde{\Lambda}\right]\right\}^{2}$ in the expression for $\delta$ calculated in Refs. [27,51].

One major effect of nonlocality is to shift the point at which the vortex sheds diffractive radiation from the edge of the shelf, $\sqrt{(x-\xi)^{2}+(y-\eta)^{2}}=\ell^{2}$, to a new radius $\tilde{\ell}$ from the vortex position $(\xi, \eta)$, which is the edge of the director response [27]. This radius for the radiation response is termed the outer-shelf radius [27]. In the present case of a finite cell, the director response extends to a boundary layer at the cell walls [34]. Hence,

$$
\tilde{\ell}=\min \left(\frac{L_{x}}{2}, \frac{L_{y}}{2}\right),
$$

and $\tilde{\Lambda}=\tilde{\ell}^{2} / 2$. For a finite cell, the diffractive radiation is then shed in a boundary layer at the cell walls.

\section{APPENDIX B: MODULATION EQUATIONS: FOURIER SERIES}

In a similar fashion to the modulation equations of Appendix A, the modulation (variational) equations for the optical vortex when the director equation (2) is solved in terms of a Fourier sine series are

$$
\begin{gathered}
\frac{d}{d z}\left[\frac{a^{2} w^{4}}{8}+g^{2} \Lambda_{1}\right]=-2 \delta \tilde{\Lambda} \kappa^{2}, \\
\sqrt{\pi} \frac{d}{d z}\left(a w^{3}\right)-4 g \Lambda_{1} \sigma^{\prime}=-2 g \Lambda_{1}\left(V_{x}^{2}+V_{y}^{2}\right)+2 g \Lambda_{2}, \\
\xi^{\prime}=V_{x}, \quad \eta^{\prime}=V_{y}, \\
\frac{d}{d z}\left[\left(\frac{a^{2} w^{4}}{8}+g^{2} \Lambda_{1}\right) V_{x}\right]=\frac{a^{4} w^{8}}{8 v L_{x}^{2} L_{y}} \sum_{n, m=1}^{\infty} \frac{n P_{1}^{2} e^{-\gamma_{1}}}{Q_{1}} \sin \left(\frac{n \pi \xi}{L_{x}}\right) \cos \left(\frac{n \pi \xi}{L_{x}}\right) \sin ^{2}\left(\frac{m \pi \eta}{L_{y}}\right), \\
\frac{d}{d z}\left[\left(\frac{a^{2} w^{4}}{8}+g^{2} \Lambda_{1}\right) V_{y}\right]=\frac{a^{4} w^{8}}{8 v L_{x} L_{y}^{2}} \sum_{n, m=1}^{\infty} \frac{m P_{1}^{2} e^{-\gamma_{1}}}{Q_{1}} \sin ^{2}\left(\frac{n \pi \xi}{L_{x}}\right) \sin \left(\frac{m \pi \eta}{L_{y}}\right) \cos \left(\frac{m \pi \eta}{L_{y}}\right), \\
\frac{d g}{d z}=\frac{a}{\sqrt{\pi} w}-\frac{\sqrt{\pi} a^{3} w^{7}}{8 v L_{x} L_{y}} \sum_{n, m=1}^{\infty}\left[\left(1+\frac{P_{1}}{2}\right) P_{1} e^{-\gamma_{1}} \sin ^{2}\left(\frac{n \pi \xi}{L_{x}}\right) \sin ^{2}\left(\frac{m \pi \eta}{L_{y}}\right)\right]-2 \pi^{3 / 2} \delta g,
\end{gathered}
$$




$$
\begin{aligned}
\frac{d \sigma}{d z}= & \frac{1}{2}\left(V_{x}^{2}+V_{y}^{2}\right)-\frac{4}{w^{2}}+\frac{\pi a^{2} w^{6}}{4 v L_{x} L_{y}} \sum_{n, m=1}^{\infty}\left[\left(1+\frac{P_{1}}{2}\right) P_{1} e^{-\gamma_{1}} \sin ^{2}\left(\frac{n \pi \xi}{L_{x}}\right) \sin ^{2}\left(\frac{m \pi \eta}{L_{y}}\right)\right] \\
& +\frac{a^{2} w^{4}}{\pi v L_{x} L_{y}} \sum_{n, m=1}^{\infty} \frac{P_{1}^{2} e^{-\gamma_{1}}}{Q_{1}} \sin ^{2}\left(\frac{n \pi \xi}{L_{x}}\right) \sin ^{2}\left(\frac{m \pi \eta}{L_{y}}\right) .
\end{aligned}
$$

Here,

$$
P_{1}=2-\gamma_{1}, \quad Q_{1}=\frac{n^{2}}{L_{x}^{2}}+\frac{m^{2}}{L_{y}^{2}}, \quad \gamma_{1}=\frac{1}{4} \pi^{2} w^{2} Q_{1} .
$$

The advantage of using the method of images over Fourier series to solve the director equation can be seen by comparing these modulation equations with those of Appendix A.

\section{APPENDIX C: MODULATION EQUATIONS: STABILITY, METHOD OF IMAGES}

$$
\begin{aligned}
& \frac{d}{d z}\left[\frac{a^{2} w^{4}}{8}+g^{2} \Lambda_{1}\right]=0 \\
& \sqrt{\pi}\left(a w^{3}\right)_{z}+2 \sqrt{\pi}(a w)_{\phi}=4 g \Lambda_{1}\left(\sigma_{z}-V_{x} \xi_{z}-V_{y} \eta_{z}+\frac{V_{x}^{2}}{2}+\frac{V_{y}^{2}}{2}\right)-2 \Lambda_{2}\left(g_{\phi \phi}-g\right), \\
& \frac{d \xi}{d z}=V_{x}, \quad \frac{d \eta}{d z}=V_{y}, \\
& \frac{d}{d z}\left[\left(\frac{a^{2} w^{4}}{8}+g^{2} \Lambda_{1}\right) V_{x}\right]=\frac{a^{4} w^{8}}{32 \nu \xi}, \\
& \frac{d}{d z}\left[\left(\frac{a^{2} w^{4}}{8}+g^{2} \Lambda_{1}\right) V_{y}\right]=0 \\
& \sqrt{\pi} \frac{d g}{d z}=\frac{a}{w}+\frac{a w_{\phi}^{2}}{w^{3}}+\frac{2 \sqrt{\pi} g_{\phi}}{w^{2}}-\frac{a_{\phi \phi}}{2 w}-\frac{a^{3} w^{5}}{16 v}, \\
& \frac{d \sigma}{d z}=\frac{1}{2}\left(V_{x}^{2}+V_{y}^{2}\right)-\frac{4}{w^{2}}-\frac{3 w_{\phi}^{2}}{w^{4}}+\frac{2 a_{\phi} w_{\phi}}{a w^{3}}-\frac{8 \sqrt{\pi} g_{\phi}}{a^{2} w^{3}}+\frac{2 a_{\phi \phi}}{a w^{2}}+\frac{w_{\phi \phi}}{w^{3}}-\frac{a^{2} w^{4}}{2 v}\left(\ln \frac{w}{\sqrt{2}}+\frac{1-2 \gamma}{4}-\ln 2 \xi\right) .
\end{aligned}
$$

[1] J. F. Nye and M. V. Berry, Proc. R. Soc. London A 336, 165 (1974).

[2] D. N. Neshev, T. J. Alexander, E. A. Ostrovskaya, Y. S. Kivshar, H. Martin, I. Makasyuk, and Z. Chen, Phys. Rev. Lett. 92, 123903 (2004).

[3] J. W. Fleischer, G. Bartal, O. Cohen, O. Manela, M. Segev, J. Hudock, and D. N. Christodoulides, Phys. Rev. Lett. 92, 123904 (2004).

[4] V. Tikhonenko, J. Christou, and B. Luther-Daves, J. Opt. Soc. Am. B 12, 2046 (1995).

[5] I. V. Basistiy, V. Yu. Bazhenov, M. S. Soskin, and M. V. Vasnetsov, Opt. Commun. 103, 422 (1993).

[6] T. J. Alexander, E. A. Ostrovskaya, Y. S. Kivshar, and P. S. Julienne, J. Opt. B 4, S33 (2002).

[7] G. Assanto, M. Peccianti, and C. Conti, Opt. Photon. News 14, 44 (2003).

[8] G. Assanto, A. Fratalocchi, and M. Peccianti, Opt. Express 15, 5248 (2007).

[9] I. C. Khoo, Phys. Rep. 471, 221 (2009).

[10] M. Peccianti and G. Assanto, Phys. Rep. 516, 147 (2012).

[11] Yu. S. Kivshar and G. Agrawal, Optical Solitons: From Fibers to Photonic Crystals (Academic, San Diego, 2003).
[12] C. Conti, M. Peccianti, and G. Assanto, Phys. Rev. Lett. 91, 073901 (2003).

[13] A. I. Yakimenko, Y. A. Zaliznyak, and Y. S. Kivshar, Phys. Rev. E 71, 065603(R) (2005).

[14] A. A. Minzoni, N. F. Smyth, A. L. Worthy, and Y. S. Kivshar, Phys. Rev. A 76, 063803 (2007).

[15] F. Lenzini, S. Residori, F. T. Arecchi, and U. Bortolozzo, Phys. Rev. A 84, 061801(R) (2011).

[16] N. F. Smyth and W. Xia, J. Phys. B 45, 165403 (2012).

[17] A. A. Minzoni, L. W. Sciberras, N. F. Smyth, and A. L. Worthy, in Nematicons: Spatial Optical Solitons in Nematic Liquid Crystals, edited by G. Assanto (Wiley, Hoboken, New Jersey, 2012).

[18] A. A. Minzoni, N. F. Smyth, and Z. Xu, Phys. Rev. A 81, 033816 (2010).

[19] Ya. V. Izdebskaya, A. S. Desyatnikov, G. Assanto, and Yu. S. Kivshar, Opt. Express 19, 21457 (2011).

[20] Ya. V. Izdebskaya, J. Rebling, A. S. Desyatnikov, and Y. S. Kivshar, Opt. Lett. 37, 767 (2012).

[21] M. Shen, J. J. Zheng, Q. Kong, Y. Y. Lin, C. C. Jeng, R. K. Lee, and W. Krolikowski, Phys. Rev. A 86, 013827 (2012).

[22] M. Padgett, J. Courtial, L. Allen, S. Franke-Arnold, and S. M. Barnett, J. Mod. Opt. 49, 777 (2002). 
[23] I. C. Khoo, Phys. Rev. A 25, 1636 (1982).

[24] I. C. Khoo, Liquid Crystals: Physical Properties and Nonlinear Optical Phenomena (Wiley, New York, 1995).

[25] A. Alberucci and G. Assanto, J. Opt. Soc. Am. B 24, 2314 (2007).

[26] A. Alberucci, G. Assanto, D. Buccoliero, A. S. Desyatnikov, T. R. Marchant, and N. F. Smyth, Phys. Rev. A 79, 043816 (2009).

[27] A. A. Minzoni, N. F. Smyth, and A. L. Worthy, J. Opt. Soc. Am. B 24, 1549 (2007).

[28] B. D. Skuse and N. F. Smyth, Phys. Rev. A 79, 063806 (2009).

[29] C. García Reimbert, A. A. Minzoni, T. R. Marchant, N. F. Smyth, and A. L. Worthy, Physica D 237, 1088 (2008).

[30] B. D. Skuse and N. F. Smyth, Phys. Rev. A 77, 013817 (2008).

[31] M. Peccianti, A. De Rossi, G. Assanto, A. De Luca, C. Umeton, and I. C. Khoo, Appl. Phys. Lett. 77, 7 (2000).

[32] M. Peccianti, C. Conti, G. Assanto, A. De Luca, and C. Umeton, Nature (London) 432, 733 (2004).

[33] A. Alberucci, M. Peccianti, and G. Assanto, Opt. Lett. 32, 2795 (2007).

[34] A. A. Minzoni, L. W. Sciberras, N. F. Smyth, and A. L. Worthy, Phys. Rev. A 84, 043823 (2011).

[35] G. B. Whitham, Linear and Nonlinear Waves (Wiley, New York, 1974).

[36] G. Assanto, A. A. Minzoni, N. F. Smyth, and A. L. Worthy, Phys. Rev. A 82, 053843 (2010).

[37] G. Assanto, A. A. Minzoni, M. Peccianti, and N. F. Smyth, Phys. Rev. A 79, 033837 (2009).

[38] G. Assanto, N. F. Smyth, and W. Xia, Phys. Rev. A 84, 033818 (2011).

[39] M. Peccianti, A. Dyadyusha, M. Kaczmarek, and G. Assanto, Nature Phys. 2, 737 (2006).
[40] M. Peccianti, G. Assanto, A. Dyadyusha, and M. Kaczmarek, Phys. Rev. Lett. 98, 113902 (2007).

[41] M. Peccianti, G. Assanto, A. Dyadyusha, and M. Kuczmarek, Opt. Lett. 32, 271 (2007).

[42] M. Peccianti, K. A. Brzdakiewicz, and G. Assanto, Opt. Lett. 27, 1460 (2002).

[43] M. Peccianti, A. Fratalocchi, and G. Assanto, Opt. Express 12, 6524 (2004).

[44] W. Wan, S. Jia, and J. W. Fleischer, Nature Phys. 3, 46 (2007).

[45] W. Wan, D. V. Dylov, C. Barsi, and J. W. Fleischer, OSA/CLEO/IQEC 2009 (IEEE, New York, 2009).

[46] E. A. Kuznetsov and A. M. Rubenchik, Phys. Rep. 142103 (1986)

[47] C. Rotschild, M. Segev, Z. Xu, Y. V. Kartashov, L. Torner, and O. Cohen, Opt. Lett. 31, 3312 (2006).

[48] C. Rotschild, B. Alfassi, O. Cohen, and M. Segev, Nature Phys. 2, 769 (2006).

[49] W. L. Kath and N. F. Smyth, Phys. Rev. E 51, 1484 (1995).

[50] J. Yang, Stud. Appl. Math. 98, 61 (1997).

[51] C. García Reimbert, A. A. Minzoni, and N. F. Smyth, J. Opt. Soc. Am. B 23, 294 (2006).

[52] R. Courant and D. Hilbert, Methods of Mathematical Physics Vol. 1 (Interscience, New York, 1965).

[53] J. Yang, New J. Phys. 6, 47 (2004).

[54] A. Alberucci, A. Piccardi, M. Peccianti, M. Kaczmarek, and G. Assanto, Phys. Rev. A 82, 023806 (2010).

[55] G. Assanto, B. D. Skuse, and N. F. Smyth, Photon. Lett. Poland 1, 154 (2009).

[56] G. Assanto, B. D. Skuse, and N. F. Smyth, Phys. Rev. A 81, 063811 (2010).

[57] G. Assanto, C. García-Reimbert, A. A. Minzoni, N. F. Smyth, and A. L. Worthy, Physica D 240, 1213 (2011). 